\title{
Generation rate scaling: the quality factor optimization of microring resonators for photon-pair sources
}

Guo, Kai; Shi, Xiaodong; Wang, Xiaolin; Yang, Junbo; Ding, Yunhong; Ou, Haiyan; Zhao, Yijun

Published in:

Photonics Research

Link to article, DOI:

10.1364/PRJ.6.000587

Publication date:

2018

Document Version

Publisher's PDF, also known as Version of record

Link back to DTU Orbit

Citation (APA):

Guo, K., Shi, X., Wang, X., Yang, J., Ding, Y., Ou, H., \& Zhao, Y. (2018). Generation rate scaling: the quality factor optimization of microring resonators for photon-pair sources. Photonics Research, 6(6), 587-596. https://doi.org/10.1364/PRJ.6.000587

\section{General rights}

Copyright and moral rights for the publications made accessible in the public portal are retained by the authors and/or other copyright owners and it is a condition of accessing publications that users recognise and abide by the legal requirements associated with these rights.

- Users may download and print one copy of any publication from the public portal for the purpose of private study or research.

- You may not further distribute the material or use it for any profit-making activity or commercial gain

- You may freely distribute the URL identifying the publication in the public portal 


\title{
Generation rate scaling: the quality factor optimization of microring resonators for photon-pair sources
}

\author{
Kal Guo, ${ }^{1, *}$ Xiaodong Shl, ${ }^{2}$ Xiaolin Wang, ${ }^{1}$ Junbo Yang, ${ }^{3}$ Yunhong Ding, ${ }^{2}$ Haiyan Ou, ${ }^{2}$ and YiJun Zhao \\ ${ }^{1}$ College of Advanced Interdisciplinary Studies, National University of Defense Technology, Changsha 410073, China \\ ${ }^{2}$ Department of Photonics Engineering, Technical University of Denmark, Kgs. Lyngby 2800, Denmark \\ ${ }^{3}$ Center of Material Science, College of Liberal Arts and Sciences, National University of Defense Technology, Changsha 410073, China \\ ${ }^{*}$ Corresponding author: guokai07203@hotmail.com
}

Received 17 January 2018; revised 27 March 2018; accepted 29 March 2018; posted 4 April 2018 (Doc. ID 320070$) ;$ published 23 May 2018

\begin{abstract}
To achieve photon-pair generation scaling, we optimize the quality factor of microring resonators for efficient continuous-wave-pumped spontaneous four-wave mixing. Numerical studies indicate that a high intrinsic quality factor makes high pair rate and pair brightness possible, in which the maximums take place under overcoupling and critical-coupling conditions, respectively. We fabricate six all-pass-type microring resonator samples on a silicon-on-insulator chip involving gap width as the only degree of freedom. The signal count rate, pair brightness, and coincidence rate of all the samples are characterized, which are then compared with the modified simulations by taking the detector saturation and nonlinear loss into account. Being experimentally validated for the first time to the best of our knowledge, this work explicitly demonstrates that reducing the round-trip loss in a ring cavity and designing the corresponding optimized gap width are more effective to generate high-rate or high-brightness photon pairs than the conventional strategy of simply increasing the quality factor. () 2018 Chinese Laser Press
\end{abstract}

OCIS codes: (190.4380) Nonlinear optics, four-wave mixing; (270.0270) Quantum optics; (190.4360) Nonlinear optics, devices.

\section{INTRODUCTION}

A quantum-correlated photon-pair source is a key resource in research of quantum optics such as quantum information processing [1] and quantum communication [2]. Thereinto, the most mature technology is quantum key distribution (QKD), where the nature of correlated photon pairs is applied to suffice high-security communication [3-7]. Sources capable of QKD are required to emit single photons in a probabilistic manner with low noise, preferably in the telecom wavelength range, to benefit from the compatibility of optical fiber networks [8]. Moreover, the single photon generated from spontaneous nonlinear processes has a naturally correlated twin photon, which makes it possible to apply the detection of one photon (signal) to herald the existence of the other (idler). While the initially heralded photon-pair sources have been demonstrated via spontaneous downconversion in optical crystals [9] or quasi-matched waveguides [10], and via spontaneous four-wave mixing (SpFWM) in optical fibers [8,11], a number of experiments were carried out in the past decade via SpFWM in integrated waveguide platforms, of which the material can be crystalline silicon [12-14], amorphous silicon [15], silica [16], silicon nitride [17], and AlGaAs [18]. Integrated waveguides often have large refractive index contrast leading to strong light confinement and high nonlinear interaction, which enables efficient photon-pair generation within a few millimeters. Moreover, by employing either butt-coupled [19] or verticalcoupled approaches [20], integrated waveguides are compatible with fiber-based systems; thus, it can be applied as the nonlinear medium of photon-pair sources instead of optical fibers, to avoid broadband spontaneous Raman scattering noise [11]. In addition, thanks to the mature fabrication methods of semiconductors and integrated circuits, which enable a variety of functionalities [21], efficient quantum communication systems are in the progress of on-chip integration [22,23].

Although photon-pair sources using SpFWM in integrated waveguides are often driven by a pulsed pump, the continuouswave $(\mathrm{CW})$ pump, with advantages of cheaper, more stable, and especially easier on-chip integration, is also widely employed. The narrow linewidth of CW pumps gives rise to strong spectral anticorrelation and projects the generated photon pairs into a classical spectral mixture [24,25], which is desired for special applications such as time-energy entanglement [26-28], wavelength-multiplexed quantum communication [29,30], and covert quantum communication $[31,32]$. Moreover, photon-pair sources capable of long-distance quantum communication are required to have a high pair rate, which corresponds 
to vast photon pairs for cryptology coding, huge loss tolerance for long-distance transmission, and enough photon pairs for cryptology decoding. High pair brightness, corresponding to high pair rate spectral density driven by specific pump power, is also desired because a narrow photon-pair bandwidth does not only make easier entanglement-based QKD but also makes the promotion of quantum key rate possible by applying dense wavelength division multiplexing. Noteworthily, the anticorrelation for CW-pumped sources can be avoided by using narrow bandwidth filtering. However, it comes at the cost of reducing photon pairs, where the pair brightness may become lower. As shown in Ref. [14], by applying narrow-bandwidth $(0.4 \mathrm{~nm})$ filtering to a CW-pumped source using a silicon strip waveguide, the highest pair rate can reach $1.6 \times 10^{6} \mathrm{~Hz}$; however, the corresponding pair brightness of $4.0 \times 10^{5}(\mathrm{~s} \cdot \mathrm{mW} \cdot \mathrm{nm})^{-1}$ remains the same order of magnitude as that in other studies $[13,33]$. A valid approach facilitating high pair brightness is to use microring resonators (MRRs) instead of straight waveguides, which provides not only narrow-bandwidth filtering but also strong cavity enhancement. Therefore, a number of experiments were carried out using different MRR designs, which achieved 1 to 2 orders of magnitude higher pair brightness but with an ultrasmall footprint [34-41].

Although MRR has shown the capability of photon-pair generation, it lacks a normative evaluation of the waveguide design, for photon-pair generation in MRR of high pair rate and high pair brightness. Moreover, a solid understanding of the key parameters of different MRR structures, especially the quality factor, is significant, based on which the optimization may put forward an approach of generation rate scaling. The quality factor is given by [42]

$$
Q=\frac{\lambda_{\text {res }}}{\Delta \lambda},
$$

where $\lambda_{\text {res }}$ and $\Delta \lambda$ denote the resonance wavelength and its full width at half-maximum (FWHM), respectively. More specifically, for an all-pass-type MRR consisting of a bus waveguide and a ring cavity, the total quality factor obtained from the transmittance of the bus waveguide is jointly determined by the round-trip loss in the ring cavity, which is quantified by the intrinsic quality factor

$$
Q_{i}=\frac{\omega}{\alpha v_{g}}
$$

and the coupling efficiency between two components, which is quantified by the external quality factor

$$
Q_{e}=\frac{2 \omega \pi R}{|\kappa|^{2} v_{g}},
$$

where $\alpha$ denotes the round-trip loss coefficient, $v_{g}$ denotes the light group velocity in the ring cavity, $R$ denotes the radius of the ring cavity, and $\kappa$ denotes the coupling coefficient [43]. The quality factor given by Eq. (1) follows

$$
\frac{1}{Q}=\frac{1}{Q_{e}}+\frac{1}{Q_{i}} \text {. }
$$

From previous studies $[41,44,45]$, the pair rate $N_{c}$ has a third-order polynomial dependence on $Q$, which indicates the larger quality factor, the better performance. However, these studies omit the impact of round-trip loss that results in $Q=Q_{e}$ and present an approach of pair rate scaling by simply increasing the gap width $g$. Although Ref. [40] shows that $N_{c}$ has a seventh-order polynomial dependence on $Q$ by taking all types of loss into account and demonstrates good agreement between simulations and measurements, their discussion based on only one MRR does not present the potential quality factor optimization that facilitates a higher pair rate. In addition, by trading off pair rate and photon-pair bandwidth, it is valid to achieve higher pair brightness $B$.

In this paper, we demonstrate the scaling approaches of pair rate and pair brightness, respectively, by involving $Q_{e}$ and $Q_{i}$ as degrees of freedom [46]. We fabricate six all-passtype MRRs on a silicon-on-insulator (SOI) chip with different $g$ and characterize the photon-pair sources using all samples to verify the numerical predictions, taking the impact of nonlinear loss in SOI platforms and detector saturation into consideration. In the end, the future direction for efficient photon-pair generation in all types of microcavity platforms is presented.

\section{THEORETICAL APPROACHES}

Based on SpFWM in a microcavity, the pair rate $N_{c}$ is quadratic in the circling pump power $P_{c}$ [47], which is defined as

$$
P_{c}=P_{p}\left|F\left(\omega_{p}\right)\right|^{2},
$$

where $P_{p}$ denotes the incident pump power in the bus waveguide. The enhancement factor $F\left(\omega_{p}\right)$ follows

$$
\left|F\left(\omega_{p}\right)\right|^{2}=\frac{2 v_{g} Q^{2}}{\pi R \omega_{p} Q_{e}\left[1+4 Q^{2}\left(\omega_{p}-\omega_{\text {res }}\right)^{2} / \omega_{\text {res }}^{2}\right]},
$$

which reaches the maximum

$$
\left|F\left(\omega_{p}\right)\right|_{\max }^{2}=\frac{2 v_{g} Q^{2}}{\pi R \omega_{p} Q_{e}},
$$

when $\omega_{p}=\omega_{\text {res }}$, that is, the pump is on-resonance. Assume that only the signal/idler photons generated from one resonance are counted, meanwhile the pump, signal, and idler approximately have the same frequency of $\omega_{\text {res}}$; then, the pair rate in signal/idler arms is calculated through

$N_{c, s / i}=\left(\gamma P_{c} 2 \pi R\right)^{2} \int_{s / i}\left|F\left(\omega_{\text {res }}-\Delta \omega\right)\right|^{2}\left|F\left(\omega_{\text {res }}+\Delta \omega\right)\right|^{2} \mathrm{~d} \Delta \omega$,

where $\gamma$ denotes the nonlinear coefficient. By substituting Eq. (7) into Eq. (8), the pair rate in the on-resonance regime is given by

$$
N_{c, s / i}=\frac{8 v_{g}^{4} \gamma^{2} P_{p}^{2} Q_{p}^{4} Q_{s / i}^{3}}{\omega_{\mathrm{res}}^{3} \pi^{2} R^{2} Q_{e, p}^{2} Q_{e, s / i}^{2}},
$$

where $Q_{p}$ and $Q_{s / i}$ denote the total quality factor, $Q_{e, p}$ and $Q_{e, s / i}$ denote the external quality factor, corresponding to the pump and signal/idler, respectively. Furthermore, by using the definition of $B=N_{c} /\left(P_{p} \Delta \lambda\right)$ with a unit of $(s \cdot \mathrm{mW} \cdot \mathrm{nm})^{-1}$ [27], the pair brightness becomes 


$$
B_{s / i}=\frac{4 v_{g}^{4} \gamma^{2} P_{p} Q_{p}^{4} Q_{s / i}^{4}}{\omega_{\mathrm{res}}^{2} \pi^{3} R^{2} c Q_{e, p}^{2} Q_{e, s / i}^{2}} .
$$

It is common to make the approximation that $Q$ and $Q_{e}$ are both wavelength independent; hence, Eqs. (9) and (10) can be simplified as

$$
\begin{gathered}
N_{c}=\frac{8 v_{g}^{4} \gamma^{2} P_{p}^{2} Q^{7}}{\omega_{\mathrm{res}}^{3} \pi^{2} R^{2} Q_{e}^{4}}, \\
B=\frac{4 v_{g}^{4} \gamma^{2} P_{p} Q^{8}}{\omega_{\mathrm{res}}^{2} \pi^{3} R^{2} c Q_{e}^{4}},
\end{gathered}
$$

which take similar forms known from Ref. [40]. Therefore, we acquire the relations, $N_{c} \propto Q^{7} / Q_{e}^{4}$ and $B \propto Q^{8} / Q_{e}^{4}$. As Eq. (6) takes the round-trip loss of both the pump and the generated photon pairs into account, when the round-trip loss is negligible in the enhancement factor definition [44,45], the foregoing relations become $N_{c} \propto Q^{3}$ and $B \propto Q^{4}$.

By utilizing Eqs. (11) and (12), we simulate $N_{c}$ and $B$ against both $Q_{i}$ and $Q_{e}$ for a specific source using silica-cladded, allpass-type MRRs, of which the ring-cavity radius $R$ is $110 \mu \mathrm{m}$ with a cross-sectional dimension of $250 \mathrm{~nm} \times 450 \mathrm{~nm}$. Being estimated via finite-difference mode solver [48], the group velocity $v_{g}$ at a pump wavelength of $1550 \mathrm{~nm}$ is $7 \times 10^{7} \mathrm{~m} / \mathrm{s}$, and the nonlinear coefficient $\gamma$ is $300 \mathrm{~mW}^{-1}$ [49], for the fundamental transverse-electric mode. The incident pump power $P_{p}$ is $1 \mathrm{~mW}$, and we assume that the source operates in the on-resonance scheme. As shown in Fig. 1(a), for a given $Q_{e}$, an increase of $Q_{i}$ gives rise to an increase of $Q$, and finally makes $N_{c}$ higher. However, for a given $Q_{i}$, the initial increase of $Q_{e}$ makes $N_{c}$ higher until the increase of $Q_{e}^{4}$ in Eq. (11) has more of an impact than does the increase of $Q^{7}$. The black solid denotes the optimized $Q_{e}$ for a given $Q_{i}$, which facilitates the highest $N_{c}$ with a slope of 3/4, which can be as well obtained from Eq. (11), involving $Q_{e} / Q_{i}$ as a variable. Moreover, as shown in Fig. 1(b), $B$ increases with $Q_{i}$ increasing more rapidly for a given $Q_{e}$ because of the eighth-order polynomial dependence. For a given $Q_{i}$, there also exists an optimized $Q_{e}$, which facilitates the highest $B$ but has a slope of 1 instead.

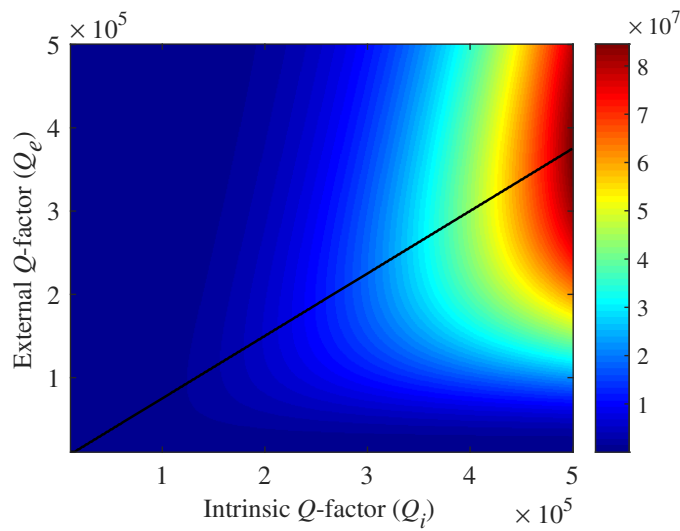

(a)
From the simulations, the primary key point of achieving high pair rate and high pair brightness is to propose high $Q_{i}$ corresponding to low $\alpha$, which is experimentally achieved by reducing the scattering loss induced by the side-wall roughness, bending loss determined by the ring-cavity radius, coupling loss determined by the gap width [50], and nonlinear loss, including two-photon absorption (TPA) and free-carrier absorption (FCA), especially in SOI platforms [51,52]. More specifically, the scattering loss is mitigated by optimizing the fabrication processes for a smoother side wall, bending loss is mitigated by designing a large ring-cavity radius, coupling loss is mitigated by designing a large gap width, and nonlinear loss is eliminated by employing specific materials such as silicon nitride and AlGaAs. Note that, when the above approaches are all used, that is, when $Q_{i}$ reaches the obtainable maximum, a suitable $Q_{e}$ is selected by designing $g$ corresponding to $\kappa$, the highest pair rate or pair brightness takes place in the overcoupling $\left(Q_{e} / Q_{i}=3 / 4\right)$ or the critical coupling $\left(Q_{e}=Q_{i}\right)$, respectively [42].

Based on the above discussion, we fabricate six MRR samples, marked as MRR1-MRR6, on the same SOI chip, involving $g$ as the single degree of freedom, designed as $180,210,240$, 270,300 , and $330 \mathrm{~nm}$, respectively. The chip was fabricated using a standard silicon-on-insulator nanofabrication processes, including e-beam lithography and inductively coupled plasma etching. Then, it was covered by $1 \mu \mathrm{m}$ thick silica cladding using plasma-enhanced chemical vapor deposition. All microring resonator samples were compactly integrated within an area of $1.8 \mathrm{~mm} \times 1.4 \mathrm{~mm}$, so the fabrication-induced difference was minimized. The free spectral range is around $0.8 \mathrm{~nm}$, which approximately matches the international telecommunication union frequency grid of $100 \mathrm{GHz}$. We measure $\Delta \lambda$ at the resonance of the pump wavelength using quite low power and derive $Q$ for each sample. We also measure the extinction ratio $\Gamma$, defined as the transmittance ratio of the minimum to the maximum that follows

$$
\Gamma=\left|\frac{Q_{e}-Q_{i}}{Q_{e}+Q_{i}}\right|^{2} .
$$

By combining Eq. (4) with Eq. (13) using the spectrum data, we estimate $Q_{e}$ and $Q_{i}$ under the assumption that $Q_{e}$

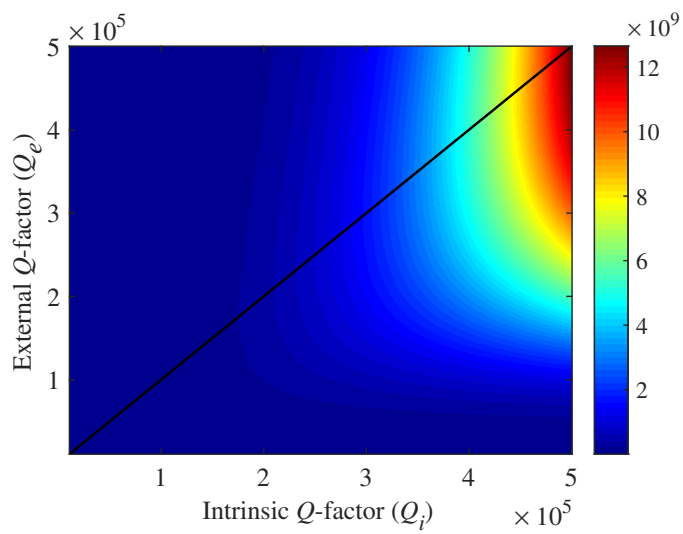

(b)

Fig. 1. (a) $N_{c}$ versus $Q_{i}$ and $Q_{e}$. Black solid denotes the optimized $Q_{e}$ for a given $Q_{i}$ that facilitates the highest $N_{c}$ with a unit of Hz. (b) $B$ versus $Q_{i}$ and $Q_{e}$. Black solid denotes the optimized $Q_{e}$ for a given $Q_{i}$ that facilitates the highest $B$ with an unit of $(s \cdot \mathrm{mW} \cdot \mathrm{nm})^{-1}$. 
Table 1. Key Parameters of MRR Samples

\begin{tabular}{|c|c|c|c|c|c|c|c|c|}
\hline Marker & $g(\mathrm{~nm})$ & $Q(P)$ & $Q_{e}(P)$ & $Q_{i}(P)$ & $Q_{e}(S)$ & $Q_{i}(S)$ & $Q_{e}(I)$ & $Q_{i}(I)$ \\
\hline MRR1 & 180 & $1.83 \times 10^{4}$ & $2.97 \times 10^{4}$ & $4.76 \times 10^{4}$ & $2.92 \times 10^{4}$ & $4.79 \times 10^{4}$ & $2.82 \times 10^{4}$ & $4.29 \times 10^{4}$ \\
\hline MRR2 & 210 & $2.59 \times 10^{4}$ & $4.83 \times 10^{4}$ & $5.59 \times 10^{4}$ & $5.23 \times 10^{4}$ & $5.35 \times 10^{4}$ & $4.94 \times 10^{4}$ & $5.81 \times 10^{4}$ \\
\hline MRR3 & 240 & $4.32 \times 10^{4}$ & $1.01 \times 10^{5}$ & $7.54 \times 10^{4}$ & $1.24 \times 10^{5}$ & $8.84 \times 10^{4}$ & $1.01 \times 10^{5}$ & $7.57 \times 10^{4}$ \\
\hline MRR5 & 300 & $7.40 \times 10^{4}$ & $2.82 \times 10^{5}$ & $1.01 \times 10^{5}$ & $2.95 \times 10^{5}$ & $9.88 \times 10^{4}$ & $2.67 \times 10^{5}$ & $1.10 \times 10^{5}$ \\
\hline MRR6 & 330 & $9.15 \times 10^{4}$ & $5.70 \times 10^{5}$ & $1.09 \times 10^{5}$ & $5.70 \times 10^{5}$ & $1.09 \times 10^{5}$ & $4.19 \times 10^{5}$ & $1.09 \times 10^{5}$ \\
\hline
\end{tabular}

and $Q_{i}$ are much higher than 1 . We used tunable-laser sweeping to measure the transmittance at low power, from which the quality factors of pump and signal/idler resonances are estimated. Because the initial resonance of each sample is detuned from the exact wavelength of pump, signal, and idler, a temperature controller was employed to achieve the on-resonance corresponding to the minimal output power. As shown in Table $1, Q_{e}$ becomes higher with $g$ increasing, which results in MRR1-MRR2 in the overcoupling and MRR3-MRR6 in the undercoupling. Moreover, only when $g$ is larger than $270 \mathrm{~nm}$ does $Q_{i}$ receive an approximate value of $1 \times 10^{5}$ because a smaller gap width brings significant coupling loss; that is, the smaller $g$, the lower $Q_{i}$.

By using the parameters in Table 1 , we calculate $N_{c}$ and $B$ for all samples by numerical simulations, according to Eqs. (9) and (10). We also simulate $N_{c}$ and $B$ versus $Q_{e}$ using $Q_{i}$ of $4.76 \times 10^{4}, 5.59 \times 10^{4}, 7.54 \times 10^{4}$, and $1.04 \times 10^{5}$. As shown in Fig. 2(a), $N_{c}$ becomes higher for MRR1-MRR4 because the increase of the $Q^{7}$ in Eq. (11) contributes more than that of the $Q_{e}^{4}$, which is more essentially attributed to the higher $Q_{i}$ corresponding to lower round-trip loss of photon pairs. For MRR4-MRR6 with approximate $Q_{i}, N_{c}$ becomes lower with $Q_{e}$ increasing because the increase of the $Q_{e}^{4}$ contributes more than that of the $Q^{7}$, which reveals the fact that both pump power coupling into the ring cavity and photon pairs coupling into the bus waveguide suffer from the low coupling efficiency. By ranking all samples in $N_{c}$ order from high to low, we predict MRR4 > MRR3 > MRR 5 > MRR2 > MRR1 > MRR6. Note that MRR6 with a higher $Q$ of $9.15 \times 10^{4}$ even generates fewer pairs than MRR1 with a lower $Q$ of $1.83 \times 10^{4}$, which contradicts the conventional understanding of using MRR with higher $Q$ for more pairs. As shown in Fig. 2(b), $B$ becomes higher for MRR1-MRR4, which is jointly contributed by the increase of $N_{c}$ and the decrease of $\Delta \lambda$, while for MRR4-MRR6, $B$ becomes lower with $Q$ increasing. By ranking all samples in $B$ order from high to low, we obtain MRR4 $>$ MRR5 $>$ MRR3 $>$ MRR6 $>$ MRR2 $>$ MRR1, where MRR6 with lower $N_{c}$ enables higher $B$ than MRR1. In addition, MRR4 with almost the highest $Q_{i}$ and the closest $Q_{e}$ to the optimized value facilitates the highest $N_{c}$ and $B$ simultaneously among all the samples, which can be potentially higher when $Q_{e}$ reduces to $7.8 \times 10^{4}$ and $1.04 \times 10^{5}$, respectively.

\section{EXPERIMENTAL CHARACTERIZATION}

We demonstrate the experimental characterization using the setup in Fig. 3. A CW laser at $1554.8 \mathrm{~nm}$ was utilized as the pump, which was power-amplified by an erbium-doped fiber amplifier (EDFA). Tunable bandpass filters (TBPFs) were applied to suppress both sideband noise and amplified spontaneous emission. A tunable attenuator (ATT) was utilized to control the incident pump power; meanwhile, the signal-tonoise ratio at a wavelength detuning of $\pm 5.6 \mathrm{~nm}$ remained $140 \mathrm{~dB}$. Photonic crystal-based grating couplers (PCGCs) [20] were used for beam-coupling between the bus waveguide and fibers, with a total insertion loss of around $12 \mathrm{~dB}$ for all samples. Input and output powers were monitored using two 99\%-1\% couplers and two power-meters (PMs) for precise $P_{p}$ estimation. Cascaded arrayed waveguide gratings (AWGs) were utilized to suppress the leaked pump field down to $-100 \mathrm{dBm}$; meanwhile, we separated the signal and idler

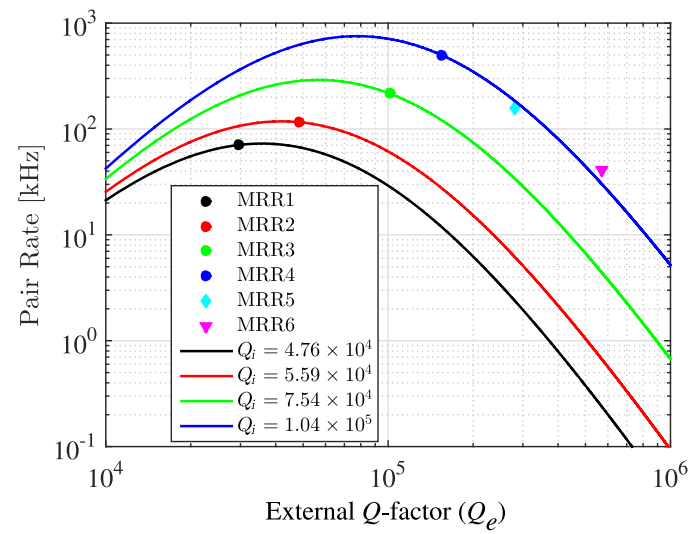

(a)

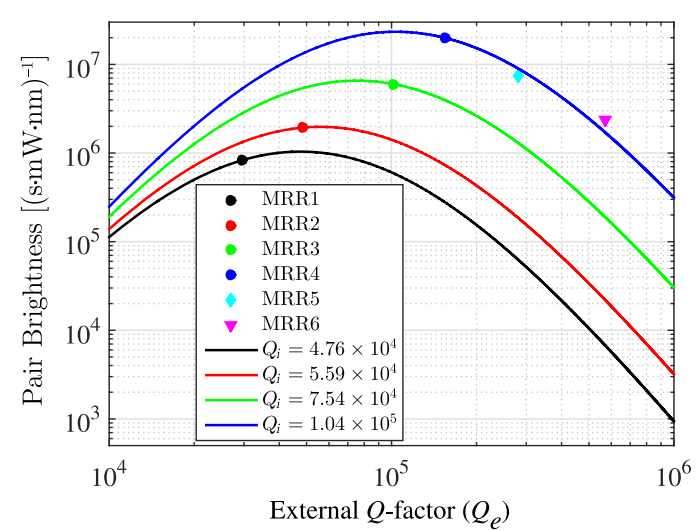

(b)

Fig. 2. (a) $N_{c}$ versus $Q_{e}$. (b) $B$ versus $Q_{e}$, when $Q_{i}$ is $4.76 \times 10^{4}$ (black solid), $5.59 \times 10^{4}$ (red solid), $7.54 \times 10^{4}$ (green solid), and $1.04 \times 10^{5}$ (blue solid). MRR1, black circle; MMR2, red circle; MRR3, green circle; MRR4, blue circle; MRR5, cyan diamond; MRR6, magenta triangle. 


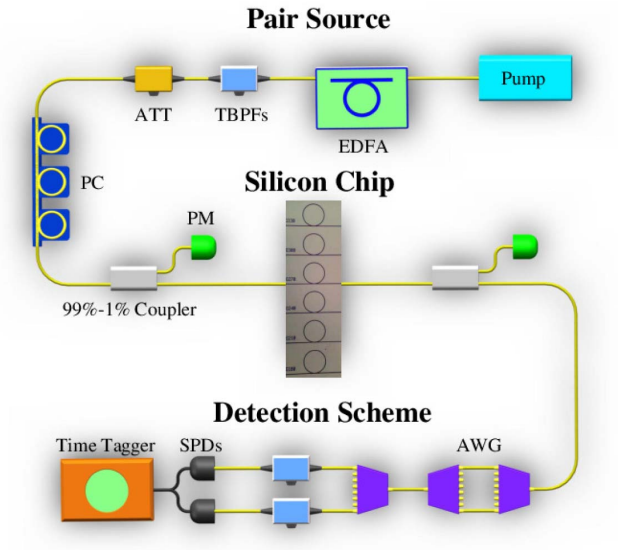

Fig. 3. Schematic of experimental setup. EDFA, erbium-doped fiber amplifier; TBPFs, tunable bandpass filters; ATT, tunable attenuator; PC, polarization controller; AWG, arrayed waveguide grating; SPD, single photon detector.

photons into two arms with wavelengths of $1549.2 \mathrm{~nm}$ and $1560.4 \mathrm{~nm}$, respectively. Through a simple calculation, undifferentiated spontaneous FWM takes place within a $3 \mathrm{~dB}$ bandwidth of $36 \mathrm{~nm}$ [49], which is much wider than the wavelength detuning in our measurement. Therefore, the measured signal/ idler count rate represents the spontaneous FWM gain under the phase-matching condition. TBPFs were utilized to further suppress the leaked pump field down to $-140 \mathrm{dBm}$, while the signal and idler photons were detected by two single-photon detectors (SPDs, ID230) in the free-running regime, with a dark count rate of $50 \mathrm{~Hz}$, dead time of $10 \mu \mathrm{s}$, and detection efficiency of $20 \%$. Finally, a time-tagging unit (ID801) was applied to calculate photon pairs.

Noteworthy, the measured signal count rate (approximating to the measured idler count rate) follows $N_{c}^{\prime}=N_{c} \eta$, where $\eta$ represents the total efficiency, including the coupling efficiency of the output PCGC, the transmittance of the after-chip components, and the detection efficiency of SPDs. As shown in Fig. $4, N_{c}^{\prime}$ in the log-log scale linearly increases with $P_{p}$ increasing and saturates at high power. The fitted slope using the

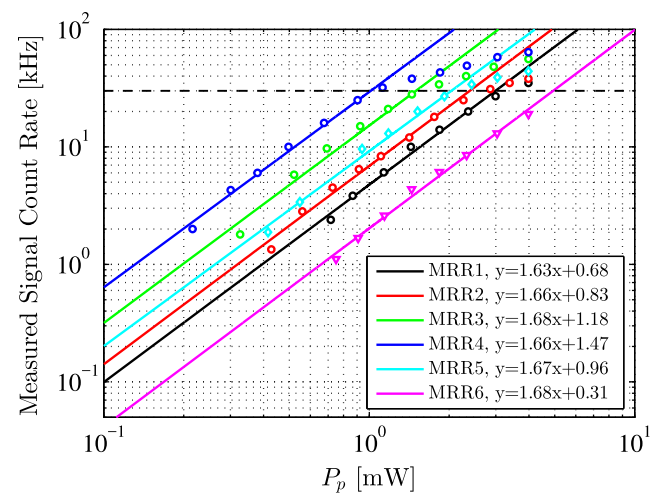

Fig. 4. $N_{c}^{\prime}$ versus $P_{p}$ for each sample. The fitted slopes are around 1.66, demonstrating that the measured photon pairs generated from SpFWM are dominant. The sequence of all samples in $N_{c}$, order from high to low, agrees well with predictions. measured signal count rate of lower than $30 \mathrm{kHz}$ (see black dash) is around 1.66 for each sample. Because $N_{c}$ is theoretically quadratic in $P_{p}$, which accounts for a slope of 2 , the measured photon pairs generated from SpFWM are dominant, especially at low power. Moreover, by taking detector saturation into account, $N_{c}^{\prime}$ can be modified through [40]

$$
N_{m c}^{\prime}=\frac{N_{c}^{\prime}-D}{1-\tau_{0} N_{c}^{\prime}},
$$

where $D$ and $\tau_{0}$ denote dark count rate and dead-time, respectively. Pumped at $3.98 \mathrm{~mW}, N_{c}^{\prime}$ for MRR1-MRR6 are measured at $35,38,56,64,44$, and $19 \mathrm{kHz}$ and modified at 54 , $61,127,178,79$, and $23 \mathrm{kHz}$, respectively, where $N_{m c}^{\prime}$ for MRR3 and MRR4 even exceed the maximal detection rate of our detector, which is $100 \mathrm{kHz}$. By applying Eq. (14) to all data, the fitted slopes increase to around 1.82, indicating that the detector saturation results in an underestimation of the measured signal count rate generated from SpFWM. Furthermore, the intrinsic nonlinear loss in SOI platforms also contributes to the signal count rate saturation because the enlarging $\alpha$ reduces $Q_{i}$ significantly at high power. The ratio of the signal count rate with nonlinear loss to that without nonlinear loss equals the ratio of $Q^{7}$ with nonlinear loss to that without nonlinear loss [40]. Hence, through a simple calculation, the maximal nonlinearloss-free $N_{m c}^{\prime}$ for each sample reaches $63,83,251,608,223$, and $53 \mathrm{kHz}$, respectively. In addition, by ranking all samples in $N_{c}^{\prime}$ (or fitted constant term) order from high to low, the result, MRR4 > MRR3 > MRR5 > MRR2 > MRR1 > MRR6, agrees well with our prediction.

As shown in Table 2, we calculate the measured pair brightness $B^{\prime}$ at $P_{p}=1 \mathrm{~mW}$ using $N_{c}^{\prime}$ and $\Delta \lambda$ for each sample, according to $B^{\prime}=N_{c}^{\prime} /\left(P_{p} \Delta \lambda\right)$. $B^{\prime}$ for MRR4 is 2 orders of magnitude higher than that for MRR1 because of smaller $\Delta \lambda$ and higher $N_{c}^{\prime}$. Although $N_{c}^{\prime}$ for MRR6 is lower than that for MRR1 and MRR2, the much smaller $\Delta \lambda$ enables $B^{\prime}$ for MRR6 to be higher. By ranking all samples in $B^{\prime}$, order from high to low, the result, MRR4 > MRR5 > MRR3 > MRR6 > MRR2 > MRR1, also agrees well with our prediction. Additionally, the saturation can also affect pair brightness, where $B^{\prime}$ for MRR4 of $6.4 \times 10^{5}(\mathrm{~s} \cdot \mathrm{mW} \cdot \mathrm{nm})^{-1}$ at $P_{p}=$ $3.98 \mathrm{~mW}$ becomes even lower than that at $P_{p}=1 \mathrm{~mW}$. By omitting detector saturation and nonlinear loss, the modified pair brightness $B_{m}^{\prime}=N_{m c}^{\prime} /\left(P_{p} \Delta \lambda\right)$ can reach $5.0 \times 10^{6}(\mathrm{~s} \cdot \mathrm{mW} \cdot \mathrm{nm})^{-1}$, which demonstrates $B \propto P_{p}$ in Eq. (12) and appears an order of magnitude higher than that shown in Ref. [14].

The coincidence rate $N_{c c}$ is of great interest, especially in the research that requires photon heralding, e.g., the time-energy entanglement [26-28]. In a temporal histogram for detection

Table 2. $N_{c}^{\prime}$ and Corresponding $B^{\prime}$ at $P_{p}=1 \mathrm{~mW}$

\begin{tabular}{lccc}
\hline Number & $\boldsymbol{N}_{\boldsymbol{c}}^{\prime}[\mathbf{k H z}]$ & $\boldsymbol{\Delta} \boldsymbol{\lambda}[\mathbf{n m}]$ & $\boldsymbol{B}^{\prime}\left[(\boldsymbol{s} \cdot \mathbf{m W} \cdot \mathbf{n m})^{-\mathbf{1}}\right]$ \\
\hline MRR1 & 4.6 & 0.085 & $5.4 \times 10^{4}$ \\
MRR2 & 6.9 & 0.060 & $1.1 \times 10^{5}$ \\
MRR3 & 15.2 & 0.036 & $4.2 \times 10^{5}$ \\
MRR4 & 30.0 & 0.025 & $1.2 \times 10^{6}$ \\
MRR5 & 9.6 & 0.021 & $4.8 \times 10^{5}$ \\
MRR6 & 2.3 & 0.017 & $1.4 \times 10^{5}$ \\
\hline
\end{tabular}


events in two arms of CW-pumped sources, the peak, corresponding to the exact time delay between the signal and idler photons, reveals $N_{c c}$, which is resolved in the SPDs' jitter time (300 ps). The average of other coincidence counts represents the accidental coincidence rate $N_{a c c}$. As shown in Figs. 5(a)-5(f), the measured $N_{c c}$ (red triangle) for all samples becomes higher with $P_{p}$ increasing because $N_{c c} \propto N_{c}$ and saturates obviously at high power. The measured $N_{c c}$ for MRR1-MRR6 at $P_{p}=3.98 \mathrm{~mW}$ reach the maximum of $14,16,23,27,16$, and $7 \mathrm{~Hz}$, respectively, which are several orders of magnitude lower than the corresponding $N_{c}$ due to $N_{c c}=N_{c} \eta_{s} \eta_{i}$. As a comparison, we present the simulation of coincidence rate (red solid), where $N_{c}$ is calculated by Eq. (9), and $\eta_{s}=\eta_{i}=0.006$ is estimated from the experiments. The simulated $N_{c c}$ is quadratic in $P_{p}$, which agrees well with the measured $N_{c c}$ at low power. However, the simulated $N_{c c}$ becomes significantly higher than the measured $N_{c c}$, where the potential maximum could reach $41,60,138,330,80$, and $23 \mathrm{~Hz}$ for MRR1-MRR6, respectively. The disagreement between simulation and measurement at high power is primarily because the general model does not take detector saturation and nonlinear loss into account. By introducing detector saturation using Eq. (14) into $N_{c}$ estimation, the modified $N_{c c}$ (red dotted) overlaps with the unmodified simulation at low power and meanwhile partly saturates at high power. At $P_{p}=3.98 \mathrm{~mW}$, the simulated $N_{c c}$ with detector saturation reaches $24,32,55$, 101, 52, and $19 \mathrm{~Hz}$ for MRR1-MRR6, respectively, demonstrating that large $N_{c c}$ at high power comes at the cost of huge accidental coincidence, which may miss the true coincidence detection.

Moreover, we assume that the external quality factor does not change with pump power, thus nonlinear loss only has an impact on the intrinsic quality factor. Note that TPA and FCA depend on the circling power $P_{c}$, which describes roundtrip enhancement for both nonlinear coefficient and nonlinear loss, instead of incident power $P_{p}$. Thus, Eq. (2) in a silicon ring cavity takes the form of

$$
\alpha_{p c}=\alpha_{l}+\frac{\beta_{T} P_{c}}{A_{\text {eff }}}+6.04 \times 10^{-10} \frac{\lambda^{2} \beta_{T} P_{c}^{2} \tau}{2 \hbar \omega_{p} A_{\text {eff }}^{2}},
$$

where $\alpha_{l}$ denotes the linear loss corresponding to the intrinsic quality factor at lower power, $\beta_{T}$ denotes the TPA coefficient valued at $5.6 \times 10^{-12} \mathrm{~m} / \mathrm{W}[52], A_{\text {eff }}$ denotes the effective mode area estimated at $6 \times 10^{-14} \mathrm{~m}^{2}$ for our sample [49], and $\tau$ denotes the free carrier lifetime valued at $10 \mathrm{~ns}$ [51]. By substituting Eqs. (2), (4), (5), and (15) into Eq. (6), we get

$$
P_{p}=\frac{\pi R \omega_{p} Q_{e}}{2 v_{g}}\left[4\left(\frac{\omega_{p}-\omega_{\text {res }}}{\omega_{\text {res }}}\right)^{2}+\left(\frac{1}{Q_{e}}+\frac{\alpha_{p c} v_{g}}{\omega_{\text {res }}}\right)^{2}\right] P_{c} .
$$

By solving this quintic equation for a given $P_{p}$, the corresponding $P_{c}$ that takes nonlinear loss into account can be acquired. By applying $P_{c}$ to Eq. (15), we get ring-cavity loss and then estimate the intrinsic quality factor by Eqs. (5) and (7). Hence, the further modified $N_{c c}$ (red dashed) gets closer to the measured $N_{c c}$, which have the maximums of $21,23,27,29$, 18 , and $8 \mathrm{~Hz}$ for MRR1-MRR6, respectively. Note that $N_{c c}$ saturation induced by nonlinear loss behaves more dominantly for MRR4-MRR6 because the round-trip loss is much smaller. The resulting sequence of all samples in $N_{c c}$ order from high to low agrees well with the general model at low power but does not fit at high power because of the nonlinear loss. In addition, the slight disagreement between the modified simulation and measurement is attributed to the uncertainty induced by the pump off-resonance, the different $\eta_{s / i}$ for each sample, the

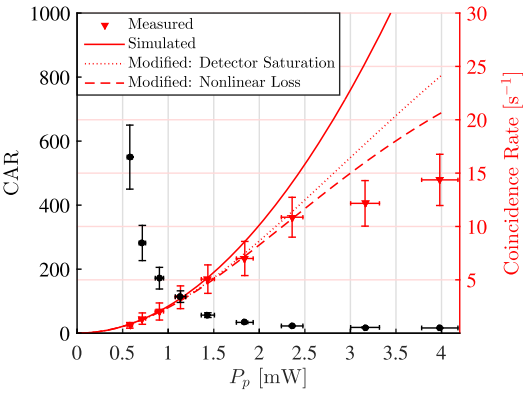

(a)

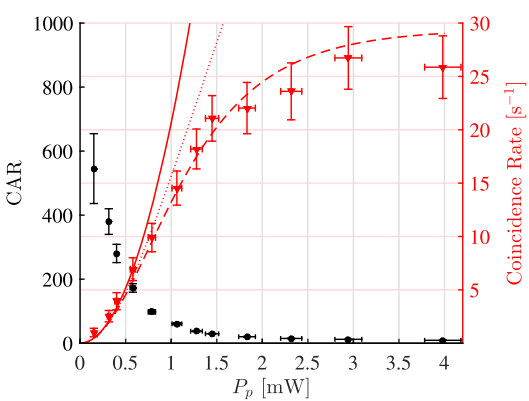

(d)

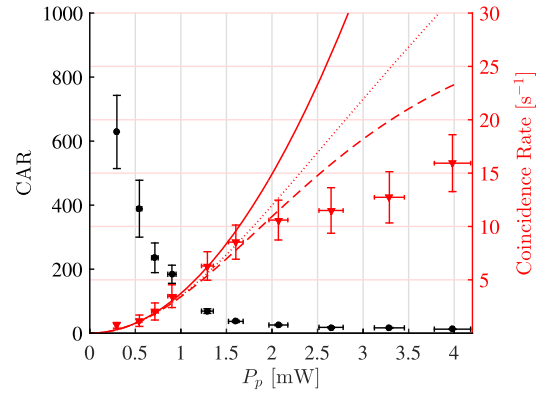

(b)

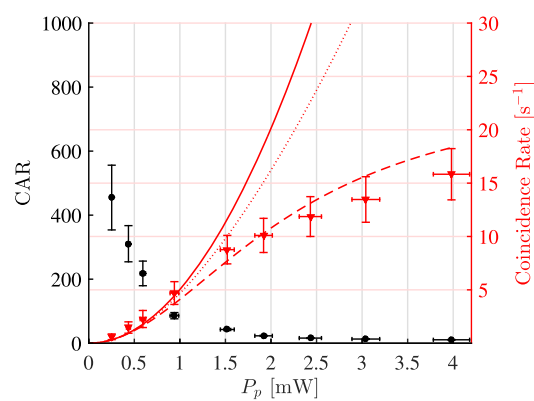

(e)

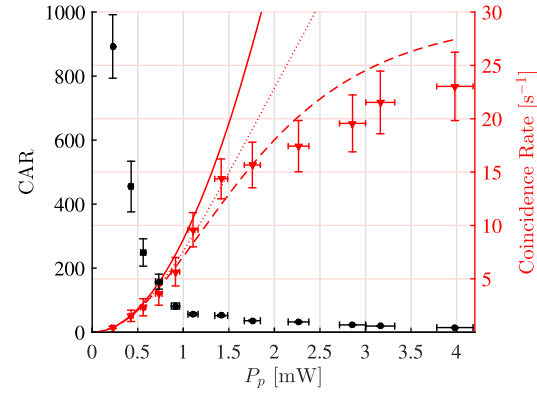

(c)

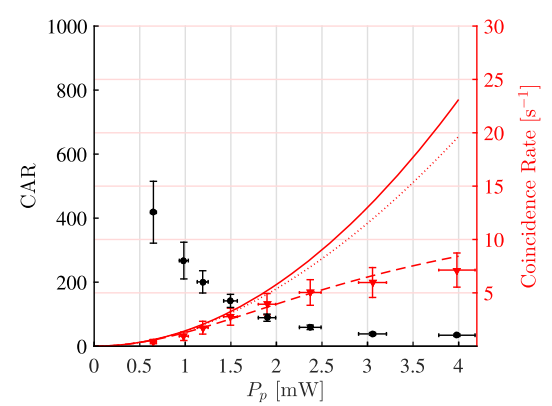

(f)

Fig. 5. $N_{c c}$ (right, red) versus $P_{p}$, including the measured data (triangle), the simulated data by Eq. (9) (solid), the modified data with detector saturation (dotted), and the modified data with nonlinear loss (dashed), and CAR versus $P_{p}$ (left, black) for (a)-(f) MRR1-MRR6. 
alignment drift during each measurement, and the slightly different parameters of each sample in calculating $N_{c}$.

Although the filtering brings insertion loss that reduces the coincidence rate, the suppression of the accidental coincidence counts is efficient. To quantify the signal-to-noise ratio of the photon pair, the coincidence-to-accidental ratio (CAR) given by

$$
\mathrm{CAR}=\frac{N_{c c}-N_{a c c}}{N_{a c c}}
$$

is measured. As shown in Figs. 5(a)-5(f) (left, black), CAR decreases with $P_{p}$ and $N_{c c}$ increasing. Because $N_{a c c} \propto$ $\left(N_{c}+N_{p n}+D / \eta_{s}\right)\left(N_{c}+N_{p n}+D / \eta_{i}\right)$, where $N_{c}$ is quadratic in $P_{p}, N_{p n}$ represents photon counts from sideband noise and the leaked pump field is linear in $P_{p}, D$ is a constant, and $N_{a c c}$ has a fourth-order polynomial dependence on $P_{p}$. At low power, $N_{a c c}$ is often 1 to 2 orders of magnitude lower than $N_{c c}$, but at high power $N_{a c c}$ almost approaches to $N_{c c}$, which greatly reduces CAR and makes it difficult to identify the true coincidence counts from the histogram. The maximal CARs for all samples are over 400 , demonstrating that all sources are operating in the low-noise regime, particularly the highest CAR of 892 , which is achieved in MRR3 with $N_{c c}$ of $0.6 \mathrm{~Hz}$.

During all experiments, a temperature controller was applied to tune the resonance wavelength such that the measurement for each sample could be carried out at on-resonance condition. Being quadratic in the circling power $P_{c}$ and further biquadratic in the enhancement factor $F$, the highest signal count rate (pair brightness, coincidence rate) takes place when the pump-resonance detuning $\lambda_{p r}$ is zero; that is, the minimal transmittance corresponds to the maximal photon pairs. Note that the FWHM of the filtering at signal/idler wavelength is larger than that of the corresponding resonance, $\lambda_{p r}$ only determines the circling pump power; then, we can use the biquadrate of the normalized enhancement factor $\left|F_{n}\left(\lambda_{p r}\right)\right|^{4}$ to describe the gain of spontaneous four-wave mixing. As shown in Figs. 6(a)-6(f), where the normalized transmittance of the pump resonance measured at quite low power is demonstrated (black solid), $N_{c c}$ at $P_{p}=3.98 \mathrm{~mW}$ (blue circle) becomes significantly higher when $\left|\lambda_{p r}\right|$ gets closer to zero, due to the dramatic increase of $\left|F_{n}\right|^{4}$ (red solid). Hence, the highest $N_{c c}$ for each sample represents the on-resonance condition and so does the signal count rate. However, the detector saturation prevents $N_{c c}$ from reaching the expected value, even when the nonlinear loss term is taken into $\left|F_{n}\right|^{4}$, which makes the on-resonance condition less important. The highest $N_{c c}$ for MRR1-MRR4 take approximate values of around 14, 16, 23, and $26 \mathrm{~Hz}$, respectively, when $\lambda_{p r}$ is in between $\pm 10 \mathrm{pm}$. Moreover, the thermal-based bistability is more obvious for the samples with a high quality factor; for example, the highest $N_{c c}$ reach $16 \mathrm{~Hz}$ and $7 \mathrm{~Hz}$ for MRR5 and MRR6, corresponding to $\lambda_{p r}=-6 \mathrm{pm}$ and $\lambda_{p r}=-4 \mathrm{pm}$, respectively. Additionally, because we have proved that MRR5-MRR6 with the higher quality factor generate less photon pairs than MRR4, it remains a challenge to achieve stable photon emission due to relatively small FWHM and huge thermal-based bistability.

To ensure that the photon pairs are generated in the singlephoton regime, we measure the heralded second-order correlation function for the source using MRR4. The photons in the idler arm pass through a 50\%-50\% coupler to be separated into two arms, $\mathrm{A}$ and $\mathrm{B}$, while the photons in the signal arm $\mathrm{H}$, are used for heralding. By carefully adjusting the fiber length in each arm to ensure the same arrival time of the photons at the detectors, the zero-delayed heralded second-order correlation function [33]

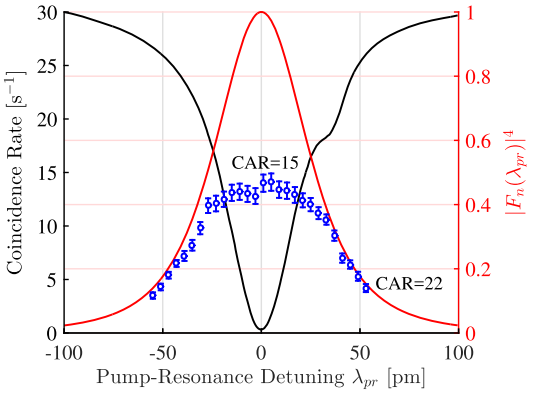

(a)

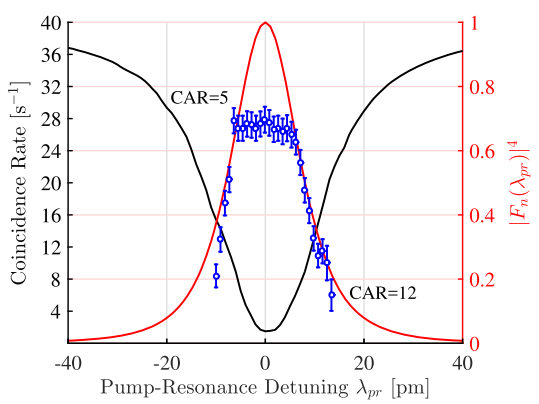

(d)

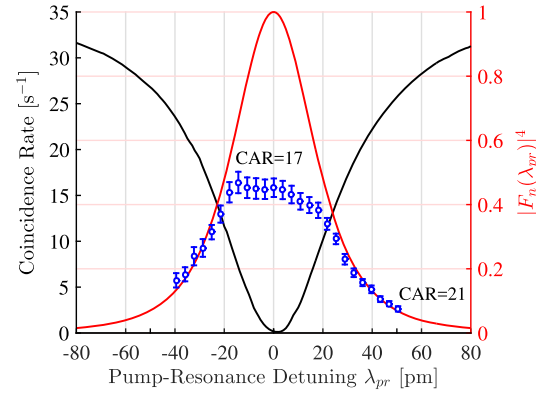

(b)

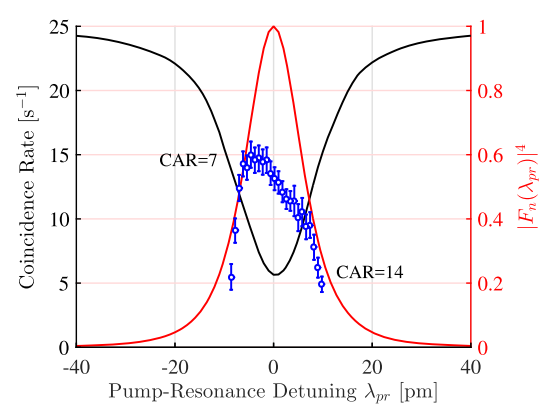

(e)

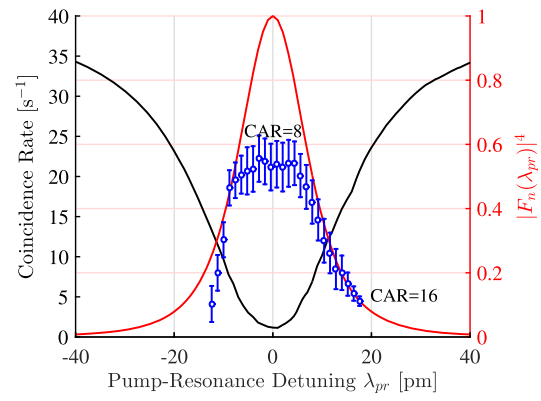

(c)

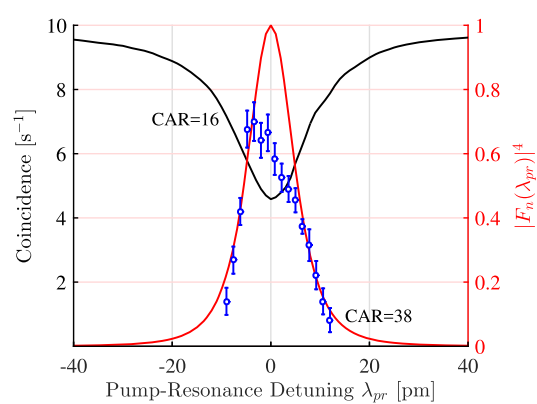

(f)

Fig. 6. (a)-(f) Normalized transmittance (black solid), biquadrate of the normalized enhancement factor $\left|F_{n}\left(\lambda_{p r}\right)\right|^{4}$ (red solid), and measured coincidence rate (blue circle), versus the pump-resonance detuning $\lambda_{p r}$ for MRR1-MRR6 at $P_{p}=3.98 \mathrm{~mW}$, respectively. 


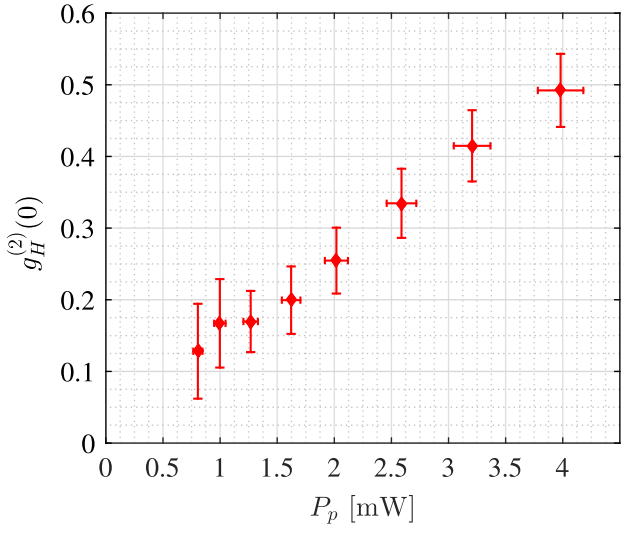

Fig. 7. Zero-delayed heralded second-order correlation $g_{H}^{(2)}(0)$ versus $P_{p}$.

$$
g_{H}^{(2)}(0)=\frac{N_{H} N_{H A B}}{N_{H A} N_{H B}}
$$

is calculated by the photon counts in $H\left(N_{H}\right)$, the coincidence counts between $H$ and $A / B\left(N_{H A} / N_{H B}\right)$, and the triple coincidence counts among all arms $\left(N_{H A B}\right)$, in a time window of 2.4 ns. As shown in Fig. $7, g_{H}^{(2)}(0)$ becomes higher with $P_{p}$ increasing, that is, the proportion of noise photons, unheralded photon pairs and heralded photon pairs operating in the multiphoton regime, becomes larger. All $g_{H}^{(2)}(0)$ of lower than 0.5 indicates that the heralded photon pairs operating in the singlephoton regime are dominant, especially at $P_{p}=0.72 \mathrm{~mW}$, where the minimal $g_{H}^{(2)}(0)$ reaches 0.13 . A lower $g_{H}^{(2)}(0)$ is expected by turning down $P_{p}$, but fewer triple coincidence counts can be detected during a long measurement. Additionally, as $N_{H A} \propto \eta_{s} \eta_{i}, N_{H B} \propto \eta_{s} \eta_{i}$, and $N_{H} \propto \eta_{s}$, it is valid to achieve lower $g_{H}^{(2)}(0)$ by increasing $\eta_{i}$, which can be achieved by using the same approach of coincidence rate scaling as previously discussed.

\section{DISCUSSION}

A general model is given to simulate the pair rate and pair brightness for photon-pair sources using spontaneous fourwave mixing in microring resonators and can be applied to the sources using other types of resonators $[28,53,54]$. The key strategy of the quality factor optimization for generation scaling is to separate the intrinsic quality factor corresponding to round-trip loss and the external quality factor corresponding to the coupling ratio. We conclude that a high intrinsic quality factor is always useful, while the external quality factor needs to be particularly designed; that is, for a given intrinsic quality factor, the highest pair rate and the highest pair brightness take place in the overcoupling regime and the critical-coupling regime, respectively. Hence, the conventional understanding of using a higher-quality factor may still be valid, but, to achieve it, special attention should be given to reduce the round-trip loss in a ring cavity instead of enlarging the gap width. From the general model, potential generation scaling can be also achieved by enlarging the nonlinear coefficient, the group velocity, or reducing the ring-cavity radius. Note that the final approach is arguable because a small radius microcavity brings large bending loss, which contradicts the intrinsic quality factor; meanwhile, its large free spectral range also limits applications, especially in quantum dense wavelength division multiplexing. We fabricate six all-pass-type microring resonators in a silicon-on-insulator chip, whose structures are the same except for the gap width, in order to characterize the photon-pair generation. The measurement demonstrates the rankings of all samples in both signal count rate and pair brightness orders from high to low that agree well with the predictions. The most explicit proof of the quality factor optimization is that the measured signal count rate ratio, by using the sample with quality factor of $9.15 \times 10^{4}$ to $6.21 \times 10^{4}$, should have been 3.18 from conventional strategies $[41,44,45]$ but becomes 0.077 in our demonstration. Thus, being experimentally validated, this work shows that reducing the round-trip loss in the ring cavity and designing a suitable gap are more effective in generation rate scaling than simply increasing the quality factor.

It is worth noting that the measured signal count rate suffers from huge saturation at high power, which is primarily attributed to the detector saturation. The saturation affects the coincidence detection strongly, which makes the on-resonance of the pump less important at high power. This issue can be improved by using the detectors with higher detection rate, shorter dead-time, and near-unity efficiency in future works [55,56]. As the modified simulation also takes nonlinear loss of silicon waveguides into account, which well describes the limitation of the coincidence rate scaling, future direction can focus on materials such as AlGaAs and silicon nitride, which have potential to achieve a high intrinsic quality factor even at high power. Moreover, the characterization can be more sufficient when the experiments are carried out in the whispering gallery mode microcavities with an ultrahigh quality factor [57-59]. The external quality factor can be continuously adjusted by moving fiber tapers [60], and the intrinsic quality factor can be flexibly controlled by transferring individual polystyrene nanoparticles [61]. More significantly for future applications, the breathtaking merits of momentum transformation ensure ultra-broadband coupling [62], and the on-chip lasing makes loss-free pump incidence possible [63]. In addition, higher total efficiency also enables a higher coincidence rate and results in a smaller non-zero-delayed heralded second-order correlation function, representing that more photon pairs operate in the lownoise single-photon scheme. To sum up, this work demonstrates the approaches of photon-pair generation scaling, especially with a suitable microring resonator design, which further benefits applications of on-chip quantum optics.

Funding. National Natural Science Foundation of China (NSFC) (60907003); Natural Science Foundation of Hunan Province, China (13JJ3001); Program for New Century Excellent Talents in University (NCET), China (NCET-120142); Danmarks Grundforskningsfond (DNRF) (DNRF123); China Scholarship Council (CSC).

Acknowledgment. The authors would like to thank Prof. Karsten Rottwitt, Dr. Davide Bacco, Dr. Mikkel Heuck, Mr. Jesper B. Christensen, Mr. Erik N. Christensen, Mr. Minghong Gao, and Mr. Dingbo Chen for their useful suggestions. 


\section{REFERENCES}

1. E. Knill, R. Laflamme, and G. J. Milburn, "A scheme for efficient quantum computation with linear optics," Nature 409, 46-52 (2001).

2. N. Gisin and R. Thew, "Quantum communication," Nat. Photonics 1, 165-171 (2007).

3. A. K. Ekert, "Quantum cryptography based on Bell's theorem," Phys. Rev. Lett. 67, 661-663 (1991).

4. C. H. Bennett, G. Brassard, and N. D. Mermin, "Quantum cryptography without Bell's theorem," Phys. Rev. Lett. 68, 557-559 (1992).

5. I. Ali-Khan, C. J. Broadbent, and J. C. Howell, "Large-alphabet quantum key distribution using energy-time entangled bipartite states," Phys. Rev. Lett. 98, 060503 (2007).

6. X. Ding, Y. He, Z. Duan, N. Gregersen, M. Chen, S. Unsleber, S. Maier, C. Schneider, M. Kamp, S. Höfling, C. Lu, and J. Pan, "Ondemand single photons with high extraction efficiency and near-unity indistinguishability from a resonantly driven quantum dot in a micropillar," Phys. Rev. Lett. 116, 020401 (2016).

7. D. Aktas, B. Fedrici, F. Kaiser, T. Lunghi, L. Labonte, and S. Tanzilli, "Entanglement distribution over $150 \mathrm{~km}$ in wavelength division multiplexed channels for quantum cryptography," Laser Photon. Rev. 10, 451-457 (2016).

8. X. Li, J. Chen, P. Voss, J. Sharping, and P. Kumar, "All-fiber photonpair source for quantum communications: improved generation of correlated photons," Opt. Express 12, 3737-3744 (2004).

9. P. G. Kwiat, K. Mattle, H. Weinfurter, A. Zeilinger, A. V. Sergienko, and Y. Shih, "New high-intensity source of polarization-entangled photon pairs," Phys. Rev. Lett. 75, 4337-4341 (1995).

10. S. Tanzilli, H. De Riedmatten, H. Tittel, H. Zbinden, P. Baldi, M. De Micheli, D. B. Ostrowsky, and N. Gisin, "Highly efficient photon-pair source using periodically poled lithium niobate waveguide," Electron. Lett. 37, 26-28 (2001).

11. S. D. Dyer, B. Baek, and S. W. Nam, "High-brightness, low-noise, allfiber photon pair source," Opt. Express 17, 10290-10297 (2009).

12. J. E. Sharping, K. F. Lee, M. A. Foster, A. C. Turner, B. S. Schmidt, M. Lipson, A. L. Gaeta, and P. Kumar, "Generation of correlated photons in nanoscale silicon waveguides," Opt. Express 14, 12388-12393 (2006).

13. K.-I. Harada, H. Takesue, H. Fukuda, T. Tsuchizawa, T. Watanabe, K. Yamada, Y. Tokura, and S.-I. Itabashi, "Frequency and polarization characteristics of correlated photon-pair generation using a silicon wire waveguide," IEEE J. Sel. Top. Quantum Electron. 16, 325-331 (2010).

14. K. Guo, E. N. Christensen, J. B. Christensen, J. G. Koefoed, D. Bacco, Y. Ding, H. Ou, and K. Rottwitt, "High coincidence-to-accidental ratio continuous-wave photon-pair generation in a grating-coupled silicon strip waveguide," Appl. Phys. Express 10, 062801 (2017).

15. S. Clemmen, A. Perret, S. Selvaraja, W. Bogaerts, D. Van Thourhout, R. Baets, P. Emplit, and S. Massar, "Generation of correlated photons in hydrogenated amorphous-silicon waveguides," Opt. Lett. 35, 3483-3485 (2010)

16. J. B. Spring, P. S. Salter, B. J. Metcalf, P. C. Humphreys, M. Moore, N. Thomas-Peter, M. Barbieri, X.-M. Jin, N. K. Langford, W. S. Kolthammer, M. J. Booth, and I. A. Walmsley, "On-chip low loss heralded source of pure single photons," Opt. Express 21, 13522-13532 (2013)

17. C. Xiong, X. Zhang, A. Mahendra, J. He, D.-Y. Choi, C. Chae, D. Marpaung, A. Leinse, R. Heideman, M. Hoekman, C. G. H. Roeloffzen, R. M. Oldenbeuving, P. W. L. van Dijk, C. Taddei, P. H. W. Leong, and B. J. Eggleton, "Compact and reconfigurable silicon nitride time-bin entanglement circuit," Optica 2, 724-727 (2015).

18. P. Kultavewuti, E. Y. Zhu, L. Qian, V. Pusino, M. Sorel, and J. S. Aitchison, "Correlated photon pair generation in ALGaAs nanowaveguides via spontaneous four-wave mixing," Opt. Express 24, 33653376 (2016).

19. M. Pu, L. Liu, H. Ou, K. Yvind, and J. M. Hvam, "Ultra-low-loss inverted taper coupler for silicon-on-insulator ridge waveguide," Opt. Commun. 283, 3678-3682 (2010)

20. Y. Ding, H. Ou, and C. Peucheret, "Ultrahigh-efficiency apodized grating coupler using fully etched photonic crystals," Opt. Lett. 38, 2732-2734 (2013).

21. J. Leuthold, C. Koos, and W. Freude, "Nonlinear silicon photonics," Nat. Photonics 4, 535-544 (2010).
22. N. C. Harris, D. Grassani, A. Simbula, M. Pant, M. Galli, T. BaehrJones, M. Hochberg, D. Englund, D. Bajoni, and C. Galland, "Integrated source of spectrally filtered correlated photons for largescale quantum photonic systems," Phys. Rev. X 4, 041047 (2014).

23. J. Wang, D. Bonneau, M. Villa, J. W. Silverstone, R. Santagati, S. Miki, T. Yamashita, M. Fujiwara, M. Sasaki, H. Terai, M. G. Tanner, C. M. Natarajan, R. H. Hadfield, J. L. O'Brien, and M. G. Thompson, "Chip-to-chip quantum photonic interconnect by path-polarization interconversion," Optica 3, 407-413 (2016).

24. I. A. Walmsley and M. G. Raymer, "Toward quantum-information processing with photons," Science 307, 1733-1734 (2005).

25. K. N. Cassemiro, K. Laiho, and C. Silberhorn, "Accessing the purity of a single photon by the width of the Hong-Ou-Mandel interference," New J. Phys. 12, 113052 (2010).

26. W. Tittel, J. Brendel, N. Gisin, and H. Zbinden, "Long-distance Belltype tests using energy-time entangled photons," Phys. Rev. A 59, 4150-4163 (1999).

27. D. Grassani, S. Azzini, M. Liscidini, M. Galli, M. J. Strain, M. Sorel, J. Sipe, and D. Bajoni, "Micrometer-scale integrated silicon source of time-energy entangled photons," Optica 2, 88-94 (2015).

28. S. Rogers, D. Mulkey, X. Lu, W. C. Jiang, and Q. Lin, "High visibility time-energy entangled photons from a silicon nanophotonic chip," ACS Photon. 3, 1754-1761 (2016).

29. C. Reimer, L. Caspani, M. Clerici, M. Ferrera, M. Kues, M. Peccianti, A. Pasquazi, L. Razzari, B. E. Little, S. T. Chu, D. J. Moss, and R Morandotti, "Integrated frequency comb source of heralded single photons," Opt. Express 22, 6535-6546 (2014).

30. F. Mazeas, M. Traetta, M. Bentivegna, F. Kaiser, D. Aktas, W. Zhang, C. Ramos, L. Ngah, T. Lunghi, E. Picholle, N. Belabas-Plougonven, X. Le Roux, E. Cassan, D. Marris-Morini, L. Vivien, G. Sauder, L. Labonté, and S. Tanzilli, "High-quality photonic entanglement for wavelength-multiplexed quantum communication based on a silicon chip," Opt. Express 24, 28731-28738 (2016).

31. J. M. Arrazola and V. Scarani, "Covert quantum communication," Phys. Rev. Lett. 117, 250503 (2016).

32. B. A. Bash, A. H. Gheorghe, M. Patel, J. L. Habif, D. Goeckel, D. Towsley, and S. Guha, "Quantum-secure covert communication on bosonic channels," Nat. Commun. 6, 8626 (2015).

33. A. S. Clark, C. Husko, M. J. Collins, G. Lehoucq, S. Xavier, A. De Rossi, S. Combrié, C. Xiong, and B. J. Eggleton, "Heralded single-photon source in a III-V photonic crystal," Opt. Lett. 38, 649-651 (2013).

34. S. Clemmen, K. P. Huy, W. Bogaerts, R. G. Baets, P. Emplit, and S. Massar, "Continuous wave photon pair generation in silicon-oninsulator waveguides and ring resonators," Opt. Express 17 16558-16570 (2009).

35. S. Azzini, D. Grassani, M. J. Strain, M. Sorel, L. Helt, J. Sipe, M. Liscidini, M. Galli, and D. Bajoni, "Ultra-low power generation of twin photons in a compact silicon ring resonator," Opt. Express 20, 2310023107 (2012).

36. K.-Y. Wang, V. G. Velev, K. F. Lee, A. S. Kowligy, P. Kumar, M. A. Foster, A. C. Foster, and Y.-P. Huang, "Multichannel photon-pair generation using hydrogenated amorphous silicon waveguides," Opt. Lett. 39, 914-917 (2014).

37. E. Engin, D. Bonneau, C. M. Natarajan, A. S. Clark, M. G. Tanner, R. H. Hadfield, S. N. Dorenbos, V. Zwiller, K. Ohira, N. Suzuki, H. Yoshida, N. lizuka, M. Ezaki, J. L. O'Brien, and M. G. Thompson, "Photon pair generation in a silicon micro-ring resonator with reverse bias enhancement," Opt. Express 21, 27826-27834 (2012).

38. Y. Guo, W. Zhang, S. Dong, Y. Huang, and J. Peng, "Telecom-band degenerate-frequency photon pair generation in silicon microring cavities," Opt. Lett. 39, 2526-2529 (2014).

39. R. Wakabayashi, M. Fujiwara, K.-I. Yoshino, Y. Nambu, M. Sasaki, and T. Aoki, "Time-bin entangled photon pair generation from $\mathrm{si}$ micro-ring resonator," Opt. Express 23, 1103-1113 (2015).

40. Y. Guo, W. Zhang, N. Lv, Q. Zhou, Y. Huang, and J. Peng, "The impact of nonlinear losses in the silicon micro-ring cavities on $\mathrm{CW}$ pumping correlated photon pair generation," Opt. Express 22, 2620-2631 (2014).

41. S. Azzini, D. Grassani, M. Galli, L. C. Andreani, M. Sorel, M. J. Strain, L. Helt, J. Sipe, M. Liscidini, and D. Bajoni, "From classical four-wave 
mixing to parametric fluorescence in silicon microring resonators," Opt. Lett. 37, 3807-3809 (2012)

42. W. Bogaerts, P. De Heyn, T. Van Vaerenbergh, K. De Vos, S. Kumar Selvaraja, T. Claes, P. Dumon, P. Bienstman, D. Van Thourhout, and R. Baets, "Silicon microring resonators," Laser Photon. Rev. 6, 47-73 (2012).

43. B. E. Little, S. T. Chu, H. A. Haus, J. Foresi, and J.-P. Laine, "Microring resonator channel dropping filters," J. Lightwave Technol. 15, 9981005 (1997).

44. L. Helt, Z. Yang, M. Liscidini, and J. Sipe, "Spontaneous four-wave mixing in microring resonators," Opt. Lett. 35, 3006-3008 (2010).

45. L. G. Helt, M. Liscidini, and J. E. Sipe, "How does it scale? Comparing quantum and classical nonlinear optical processes in integrated devices," J. Opt. Soc. Am. B 29, 2199-2212 (2012).

46. Z. Vernon, M. Liscidini, and J. Sipe, "No free lunch: the trade-off between heralding rate and efficiency in microresonator-based heralded single photon sources," Opt. Lett. 41, 788-791 (2016).

47. M. Soltani, "Novel integrated silicon nanophotonic structures using ultra-high Q resonators," Ph.D. thesis (Georgia Institute of Technology, 2009).

48. A. B. Fallahkhair, K. S. Li, and T. E. Murphy, "Vector finite difference modesolver for anisotropic dielectric waveguides," J. Lightwave Technol. 26, 1423-1431 (2008).

49. K. Guo, F. Smm, J. B. Christensen, E. N. Christensen, X. Shi, Y. Ding, H. Ou, and K. Rottwitt, "Full-vectorial propagation model and modified effective mode area of four-wave mixing in straight waveguides," Opt. Lett. 42, 3670-3673 (2017).

50. Y. Li, A. V. Maslov, N. I. Limberopoulos, A. M. Urbas, and V. N. Astratov, "Spectrally resolved resonant propulsion of dielectric microspheres," Laser Photon. Rev. 9, 263-273 (2015).

51. H. Rong, A. Liu, R. Nicolaescu, M. Paniccia, O. Cohen, and D. Hak, "Raman gain and nonlinear optical absorption measurements in a low-loss silicon waveguide," Appl. Phys. Lett. 85, 2196-2198 (2004).

52. D. Dimitropoulos, R. Jhaveri, R. Claps, J. Woo, and B. Jalali, "Lifetime of photogenerated carriers in silicon-on-insulator rib waveguides," Appl. Phys. Lett. 86, 071115 (2005).
53. X. Lu, W. C. Jiang, J. Zhang, and Q. Lin, "Biphoton statistics of quantum light generated on a silicon chip," ACS Photon. 3, 1626-1636 (2016).

54. W. C. Jiang, X. Lu, J. Zhang, O. Painter, and Q. Lin, "Silicon-chip source of bright photon pairs," Opt. Express 23, 20884-20904 (2015).

55. B. Korzh, N. Walenta, T. Lunghi, N. Gisin, and H. Zbinden, "Freerunning ingaas single photon detector with 1 dark count per second at 10\% efficiency," Appl. Phys. Lett. 104, 081108 (2014).

56. L. C. Comandar, B. Fröhlich, J. F. Dynes, A. W. Sharpe, M. Lucamarini, Z. Yuan, R. V. Penty, and A. J. Shields, "Gigahertz-gated InGaAs/InP single-photon detector with detection efficiency exceeding 55\% at $1550 \mathrm{~nm}$," J. Appl. Phys. 117, 083109 (2015).

57. Y. F. Xiao, X. F. Jiang, Q. F. Yang, L. Wang, K. Shi, Y. Li, and Q. Gong, "Tunneling-induced transparency in a chaotic microcavity," Laser Photon. Rev. 7, L51-L54 (2013).

58. X. F. Jiang, C. L. Zou, L. Wang, Q. Gong, and Y. F. Xiao, "Whisperinggallery microcavities with unidirectional laser emission," Laser Photon. Rev. 10, 40-61 (2016).

59. Y. Yang, X. Jiang, S. Kasumie, G. Zhao, L. Xu, J. M. Ward, L. Yang, and S. N. Chormaic, "Four-wave mixing parametric oscillation and frequency comb generation at visible wavelengths in a silica microbubble resonator," Opt. Lett. 41, 5266-5269 (2016).

60. M. Cai, O. Painter, and K. J. Vahala, "Observation of critical coupling in a fiber taper to a silica-microsphere whispering-gallery mode system," Phys. Rev. Lett. 85, 74-77 (2000).

61. X. F. Jiang, Y. F. Xiao, Q. F. Yang, and L. Shao, "Free-space coupled, ultralow-threshold raman lasing from a silica microcavity," Appl. Phys. Lett. 103, 101102 (2013).

62. X. Jiang, L. Shao, S. X. Zhang, X. Yi, J. Wiersig, L. Wang, Q. Gong, M. Lončar, L. Yang, and Y. F. Xiao, "Chaos-assisted broadband momentum transformation in optical microresonators," Science 358, 344-347 (2017).

63. X. F. Jiang, Y. F. Xiao, C. L. Zou, L. He, C. H. Dong, B. B. Li, Y. Li, F. W. Sun, L. Yang, and Q. Gong, "Highly unidirectional emission and ultralow-threshold lasing from on-chip ultrahigh-Q microcavities," Adv. Mater. 24, OP260-OP264 (2012). 